\title{
Situación epidemiológica de hepatitis A en Chile
}

\author{
BÁRBARA MEDINA D. ${ }^{1}$, ANDREA OLEA N. ${ }^{2}$ y XIMENA AGUILERA S. ${ }^{3}$
}

\section{Epidemiology of hepatitis A in Chile}

Chile has a intermediate endemicity pattern of hepatitis A with a progressive displacement of age disease to adolescents and adults. Since 1975 to 2003 the relative importance of children under 10 year old has diminished in $35 \%$ but a $197 \%$ increase has been observed in 15 to 24 years age group. Epidemic cycles occur each 3 to 5 years reaching rates of 80 to 100 cases/100.000 inhabitants. During 2003 and to date of this document 8.263 cases have been reported with a national cumulative rate of 52,4 cases/100.000 and maximum in 5 to 9 years $(194 / 100.000)$ and 10 to 14 years $(157 / 100.000)$ age groups. Cases distribution is all along the country, most as community outbreaks and mainly in low income groups. Since year 2002 vaccination has replaced human immunoglobulin for close contacts prophylaxis. Nowadays education campaign has been reinforced trying to stop person to person transmission and sewage treatment plants have been built to finish with the environmental long cycle of transmission.

Key words: Hepatitis A; National epidemiology; Endemic hepatitis A; Surveillance.

\section{Introducción}

\section{Transición endémica}

La hepatitis A es una enfermedad transmisible de distribución mundial. La Organización Mundial de la Salud estima que cada año ocurren en el mundo 1,5 millones de casos clínicos ${ }^{1}$. Se ha establecido que la incidencia de la hepatitis A está estrechamente relacionada al desarrollo socioeconómico y las condiciones sanitarias ${ }^{1,2}$, lo cual se reafirma por el hecho que sólo países desarrollados y con elevados niveles de vida, como los países nórdicos, han podido controlar su incidencia y tener niveles de endemia baja o casos esporádicos.

Al igual que en gran parte del mundo, la hepatitis A es endémica en nuestro país. No obstante, su patrón endémico ha evidenciado importantes cambios durante las últimas décadas, probablemente asociados a los progresos en saneamiento básico y educación que ha experimentado el país, presentando actualmente las características de endemia media. Esta presentación, típica de un período de transición entre la alta y una baja endemia, implica un desplazamiento de la enfermedad a edades mayores de la vida, afectando a niños, adolescentes y adultos jóvenes. Otra característica es la presencia de ciclos epidémicos periódicos, gatillados principalmente por la acumulación de susceptibles ${ }^{3}$.

El patrón de endemia alta se caracteriza por tasas de incidencia consistentemente elevadas, con epidemias cada 5 a 10 años, de varios años de duración y con picos de incidencia generalmente mayor a 700 casos por cien mil habitantes. Pocos casos ocurren en personas mayores de 5 años. Los datos de seroprevalencia indican que 30 a $40 \%$ de los niños adquieren la infección antes de los 5 años de edad y casi la totalidad de los adultos jóvenes poseen inmunidad ${ }^{3}$.

Por el contrario, el patrón de endemia baja se caracteriza por afectar principalmente a niños mayores y adultos jóvenes, la cadena de transmisión más frecuente es a partir del viaje a zonas de

\footnotetext{
Unidad de Vigilancia, Departamento de Epidemiología, Ministerio de Salud.

Encargada de Unidad de Vigilancia, Departamento de Epidemiología, Ministerio de Salud.

Jefe de Departamento de Epidemiología. Ministerio de Salud.
} 
alta endemia y los brotes comunitarios dispersos son infrecuentes ${ }^{3}$.

El cambio en la distribución etaria de los casos nacionales durante los últimos 28 años se observa en la Figura $1 *$. Así por ejemplo, la importancia relativa de los niños bajo 10 años de edad dentro del total de casos, ha descendido en $35 \%$ entre 1975 y 2003 (de 69 a 45\%), mientras que los adolescentes y jóvenes del grupo de 15 a 24 años han aumentado en $197 \%$ (de 5,6 a 16,6\%). Este desplazamiento de la enfermedad, que se debe a un retraso en el primer contacto con el virus, condiciona la paradoja de observar un aumento del número de casos clínicos, dado que la probabilidad de desarrollar una infección clínica, así como el riesgo de gravedad, aumenta conforme a la edad ${ }^{1}$. Por tal motivo, si bien existe una tendencia al descenso en la incidencia de hepatitis A durante las últimas décadas, ésta es discreta.

En el análisis de la tendencia de las tasas específicas de incidencia por grupos de edad, destaca el descenso sostenido en el grupo de 1 a 4 años, principal reservorio del virus, y el aumento experimentado por los grupos de 15 a 19 y 20 a 24 años (Figuras 2 y 3). La variación en los grupos de riesgo no ha sido homogénea entre las distintas regiones del país ${ }^{4}$, lo que podría ser explicado por las diferencias en las condiciones de saneamiento básico, nivel socioeconómico y acceso a educación, entre otros factores.

La mortalidad específica por esta enfermedad ha experimentado un importante descenso (Figura 4), con una letalidad que ha fluctuado entre 0,2 y $0,6 \%$ en el período 1986 - 2000, bajando a sólo $0,02 \%$ en 2001.

Los ciclos epidémicos se presentan en nuestro país cada 3 a 5 años, alcanzando tasas de incidencia máximas de entre 80 y 110 casos por cien mil habitantes, lo que corresponde a lo descrito en la literatura para comunidades de endemia intermedia ${ }^{3}$.

\section{Situación epidemiológica actual}

Desde el segundo semestre de 2002 nuestro país ha iniciado un ciclo epidémico, el que se ha caracterizado por ser menos pronunciado que los anteriores. La tasa de incidencia en 2002 fue de 70,1 por cien mil hbtes, con el doble de los casos esperados para un año no epidémico. En el plano nacional, el máximo de la epidemia se presentó en noviembre (Figura 5), marcado por el ingreso de la Región Metropolitana al ciclo, que por su magnitud de población, concentró la mayor proporción de casos. Durante el 2003 se ha mantenido el ciclo, con 8.263 casos notificados a la fecha $^{5}$. La incidencia acumulada en lo que va del año es de 52,4 casos por cien mil hbtes.

Las mayores incidencias la presentan los grupos etarios de 5 a 9 años y 10 a 14 años con 194 y 157 casos por cien mil habitantes respectivamente durante 2003. Los niños bajo 15 años de edad concentran el $72 \%$ de los casos (34\% están en el grupo de 5 a 9 años) y no existen diferen-

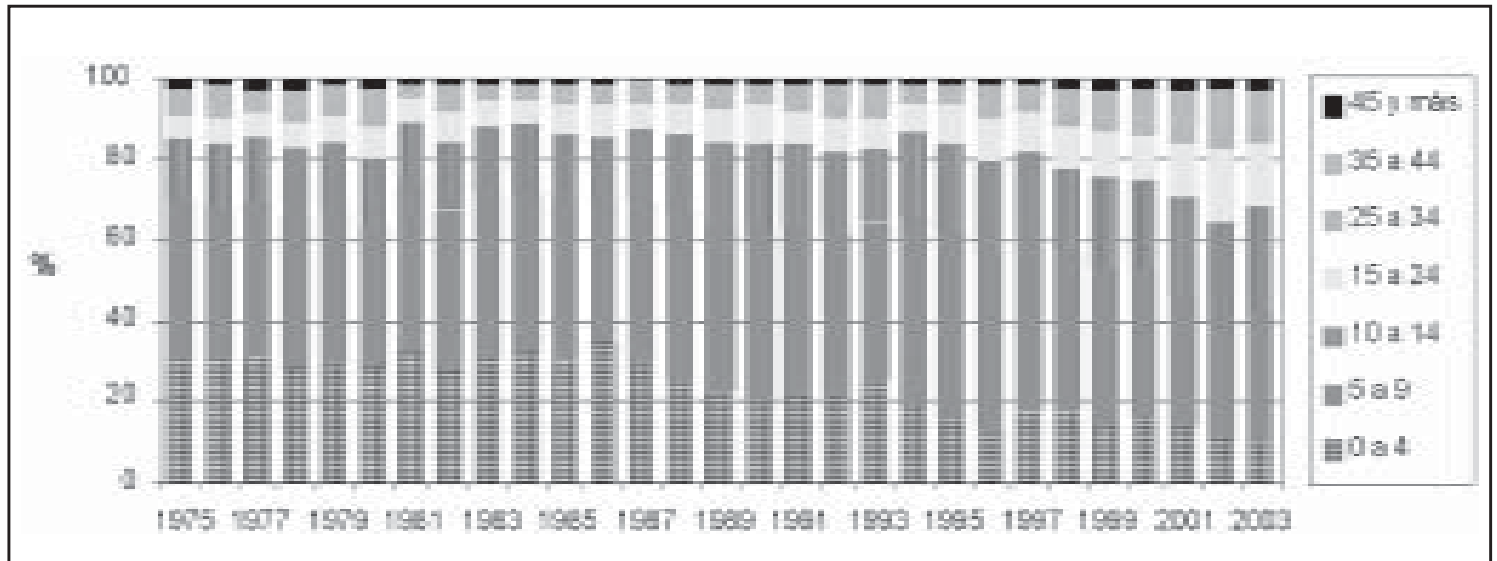

Figura 1. Distribución de los casos de hepatitis aguda viral según grupos de edad. Chile, 1975-2003. Fuente: Base de datos de ENO. Departamento de Estadísticas e Información en Salud. Ministerio de Salud. Diagnósticos utilizados: 1965-1979: hepatitis infecciosa, 1980-2001: todas hepatitis agudas excepto B, 2003-2003: hepatitis A y hepatitis aguda viral sin especificación.

\footnotetext{
* Nota metodológica: Para el análisis y gráficos de este artículo se consideraron los siguientes diagnósticos: 1975-1979: hepatitis infecciosa. 1980-2001: hepatitis aguda viral excepto B. 2002-2003: hepatitis A y hepatitis aguda viral sin especificación.
} 


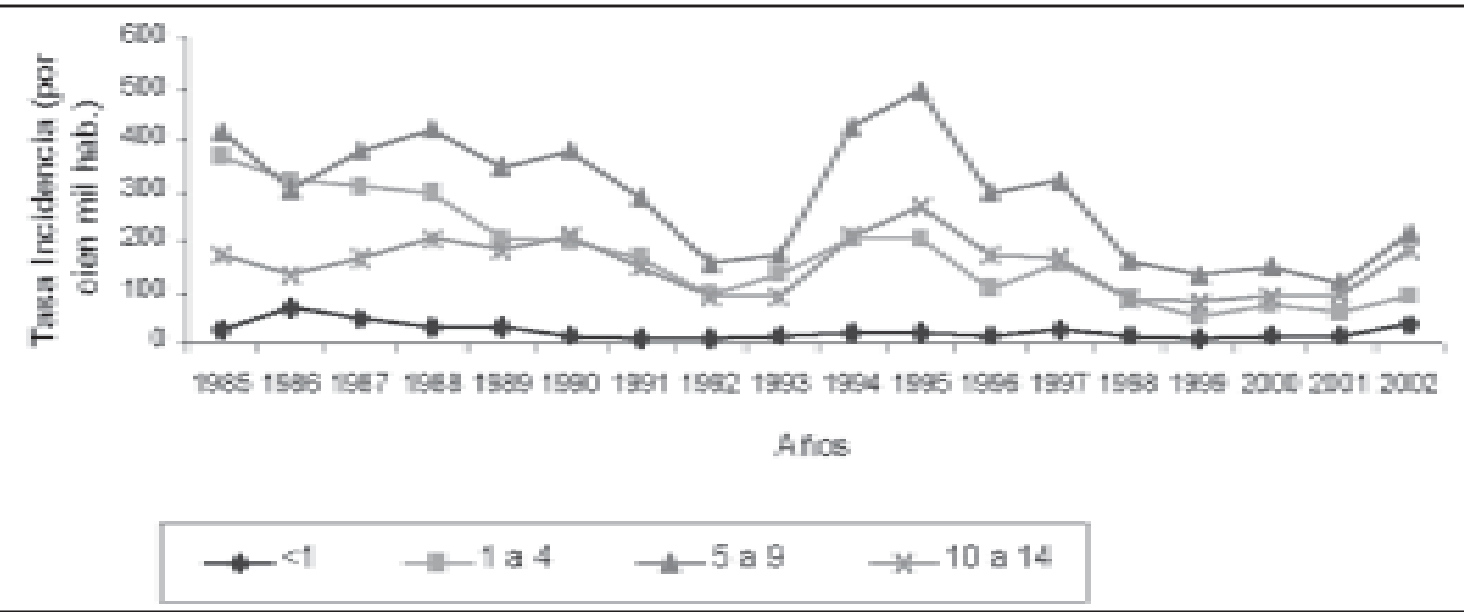

Figura 2. Tasa de incidencia de hepatitis aguda viral en grupos de edad seleccionados. Chile, 1985-2002.

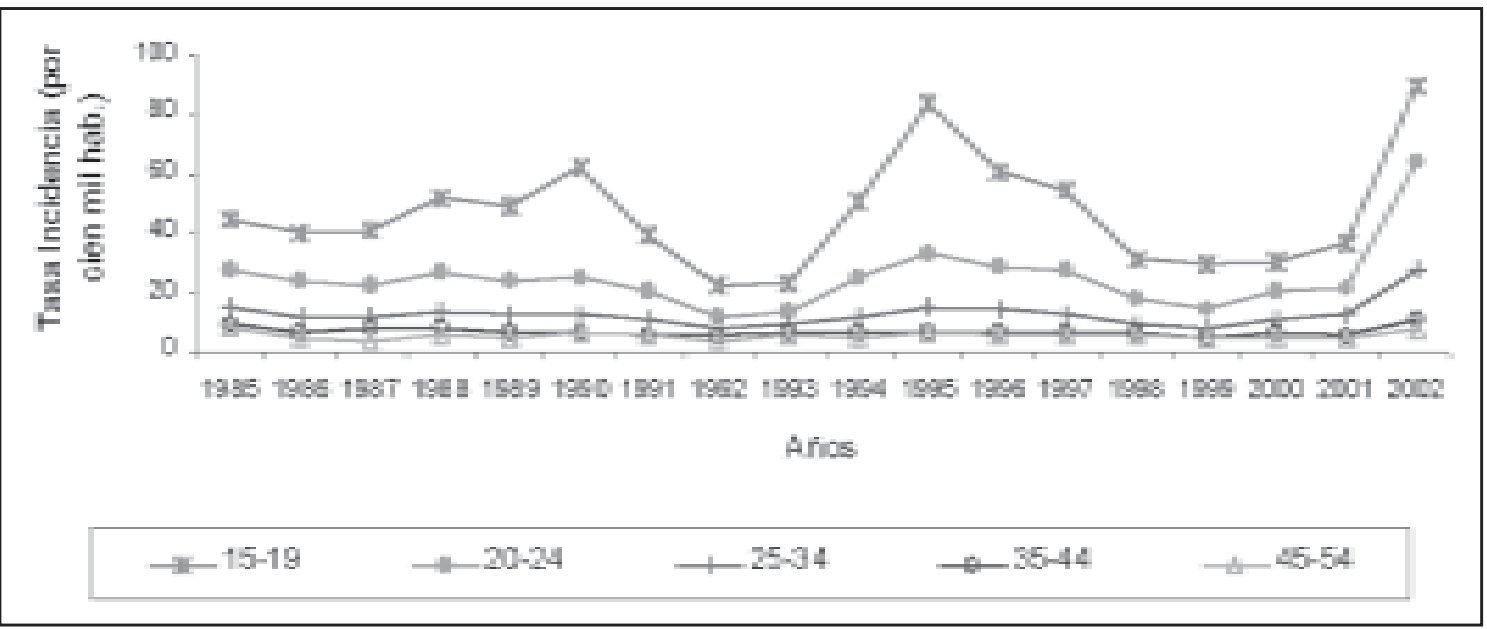

Figura 3. Tasa de incidencia de hepatitis aguda viral en grupos de edad seleccionados. Chile, 1985-2002.

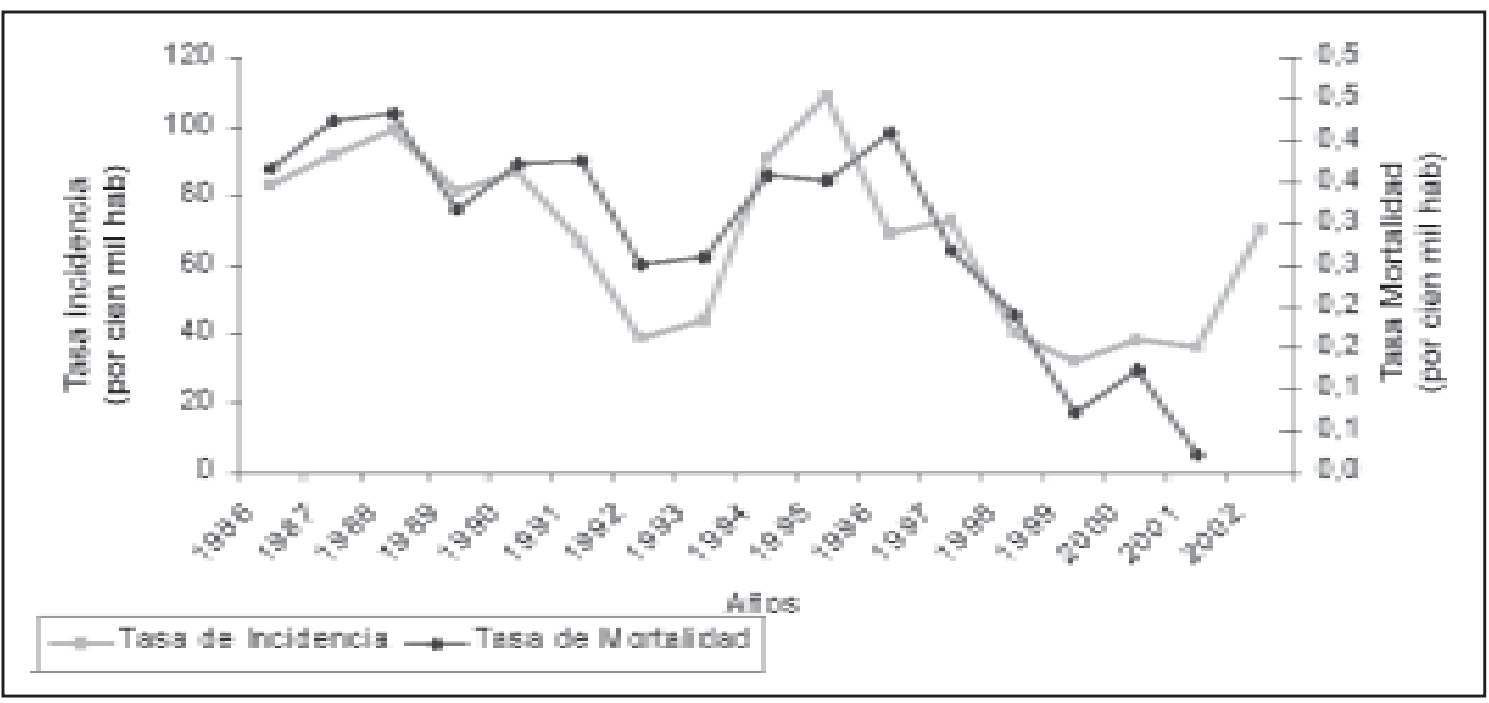

Figura 4. Tasa de incidencia y mortalidad por hepatitis A. Chile, 1986-2002. 


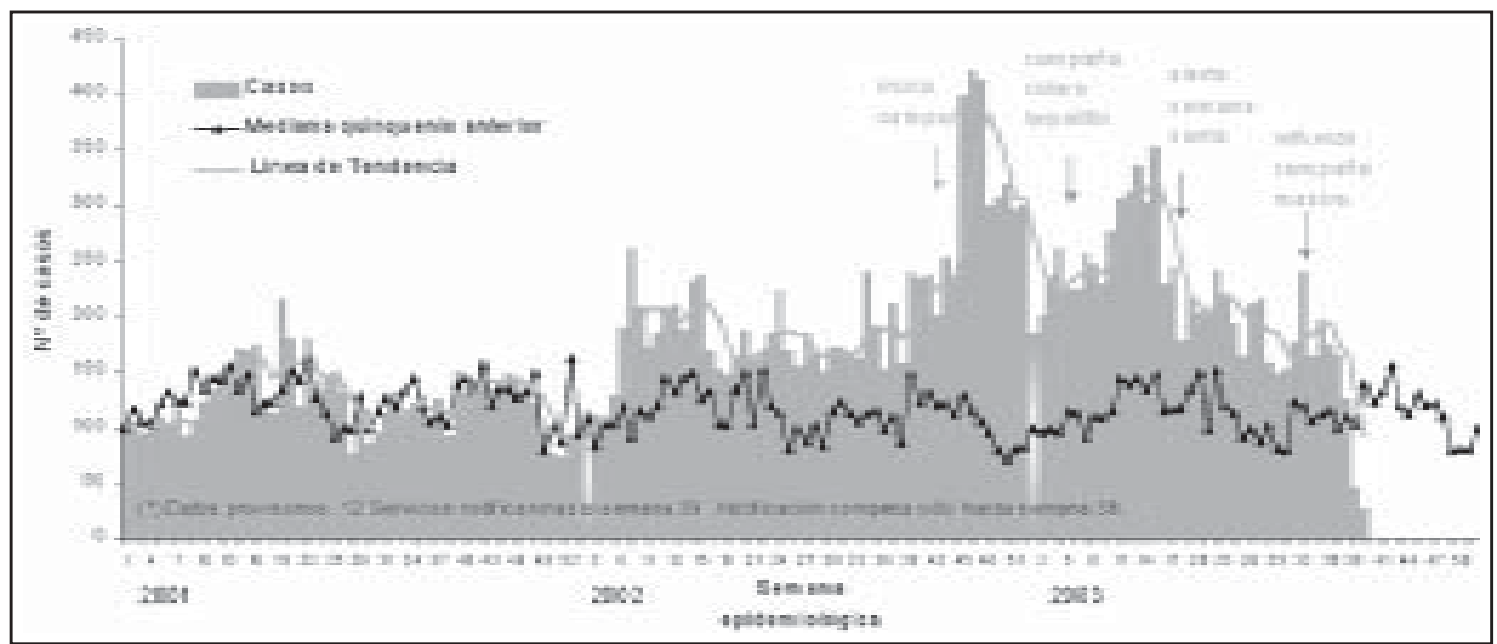

Figura 5. Curva epidémica de hepatitis A según semana de notificación. Chile 2001 - semana 39* de 2003.

cias por sexo. Sólo $0,8 \%$ de los casos han sido notificados como hepatitis A o hepatitis viral sin especificación, asociada a coma hepático.

La hepatitis A se presenta a lo largo de todo el país. Al analizar la distribución geográfica de los riesgos destaca que Iquique, Atacama, O’Higgins, Maule, Araucanía Norte y los Servicios de Salud Metropolitanos con excepción de Oriente, presentan incidencias que sobrepasan la nacional. Durante 2002, las mayores incidencias las presentaron los Servicios Atacama, Arauco, Antofagasta y Araucanía Norte (Figura 6).

Es característico de las regiones de endemia intermedia en episodios de epidemia, la presentación de brotes circunscritos en comunidades cerradas, tales como guarderías e internados, entre otros. Sin embargo, no es infrecuente la aparición de brotes comunitarios dispersos ${ }^{1,3}$. Ambas situaciones se han presentado en nuestro país durante el reciente ciclo epidémico. Desde el segundo semestre de 2002 se han notificado al Ministerio de Salud alrededor de 180 brotes de hepatitis A, con un promedio de 5 casos involucrados. El $40 \%$ de ellos se ha presentado en instituciones, principalmente colegios ${ }^{6}$. Cabe destacar que en noviembre de 2002 en Ministerio de Salud implementó el uso de vacuna anti hepatitis A para el control de brotes, con base en evidencia científica y experiencias internacionales $^{7-9}$.

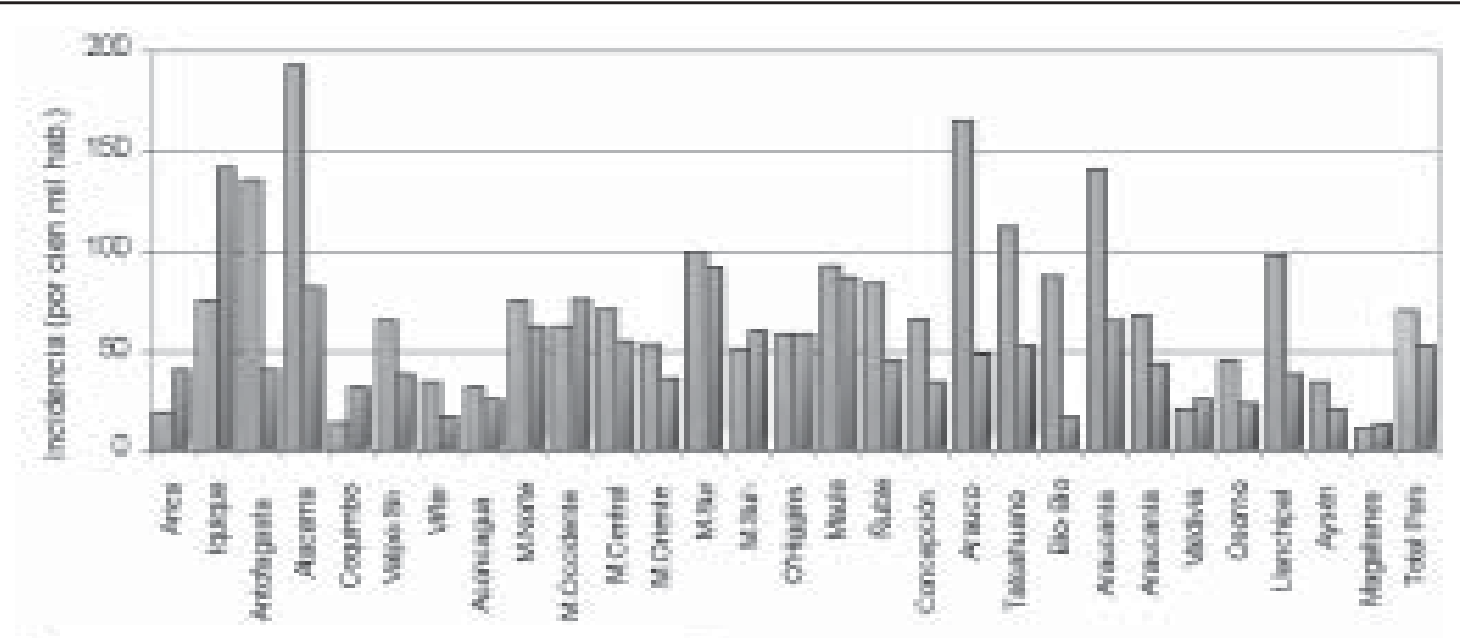

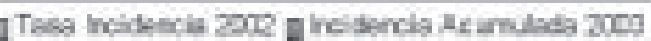

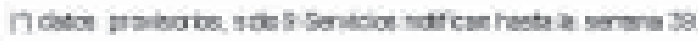

Figura 6. Tasa de incidencia de hepatitis A según Servicios de Salud. Chile, 2002 y semanas 1-39 de 2003* 
Tabla 1. Medidas adoptadas por el Ministerio de Salud para la prevención y el control de la hepatitis A

Se han orientado en 3 pilares fundamentales ${ }^{4}$ :

- Campaña comunicacional masiva y difusión en establecimientos de salud y educacionales, para reforzar en la población las medidas de prevención.

- Investigación en terreno de los brotes de hepatitis y medidas de intervención ambiental, tanto estructurales como educativas según corresponda. Coordinación intersectorial (Obras Públicas, Educación, Municipalidades) para manejo de determinantes estructurales y actividades educativas.

- Uso de vacuna anti-hepatitis A para el control de brotes. Durante 2003 el Ministerio de Salud ha adquirido 22.500 dosis de vacuna, con un costo aproximado de \$205 millones.

La tendencia actual de la enfermedad es hacia la reducción del número de casos, acercándose a la frecuencia esperada de la enfermedad y al cierre del ciclo epidémico (Figura 5). Dicha situación se observa también en la mayoría de los Servicios de Salud del país, con excepción de Iquique y Talcahuano. Iquique destaca por presentar la incidencia más alta del país, triplicando la nacional y Talcahuano, que había presentado su alza epidémica a comienzos del año 2002, muestra el inicio de un segundo ciclo desde la semana 13.

La reducción en la presentación de casos probablemente puede atribuirse a:

- El agotamiento de la población susceptible (los casos sintomáticos, casos asintomáticos y vacunados han dejado de serlo).

- El impacto de las medidas de prevención y control (Tabla 1), orientadas principalmente a cambiar las conductas de la población. Cabe destacar que la campaña comunicacional fue reforzada desde agosto pasado con el objeto de impedir el repunte primaveral.

No obstante el descenso observado a nivel nacional, debido a la estacionalidad de la enfermedad se está alerta ante un eventual rebrote primaveral, que a la fecha no se ha producido.

\section{Comentarios}

Los datos que aporta la epidemiología descriptiva de los casos notificados durante la vigilancia epidemiológica, respecto a la evolución del nivel de endemia de la hepatitis A en nuestro país, son consistentes con los hallazgos de los estudios de seroprevalencia. Estos han demostrado diferencias en distintas regiones del país y por nivel socioeconómico, además de un cambio de las prevalencia de anticuerpos protectores en las últimas décadas 2 . Así por ejemplo, en Valdivia, mientras que en 1984 el $97 \%$ de los adultos tenía anticuerpos contra el VHA, en 2002 la prevalencia de anticuerpos en adultos jóvenes (19 a 30 años) fue de $67 \%$, evidenciándose en ambos estudios transversales diferencias según nivel socioeconómico $^{10}$. Según datos aportados por diversos estudios locales, se estima que aproximadamente $60 \%$ de los adultos de nivel socioeconómico alto y 20 a $30 \%$ de los de nivel bajo son susceptibles de contraer la enfermedad ${ }^{2}$. Sin embargo, es necesario complementar esta información con un estudios de seroprevalencia en una muestra representativa de toda la población nacional, para dar mayor validez a esos hallazgos.

Los ciclos epidémicos se presentan en nuestro país cada 3 a 5 años. La duración de los períodos interepidémicos, al igual que en otras enfermedades transmisibles suele ser estable y está determinada principalmente por la magnitud de la población ${ }^{11}$. Es esperable que estos períodos se vayan prolongando y que la duración de los ciclos se reduzca al ir disminuyendo la circulación del virus.

Es importante también el rol de las medidas de prevención por parte de la población, en evitar, retrasar o controlar estos ciclos epidémicos, en el contexto de un país con importantes progresos en las coberturas de agua potable, alcantarillado y tratamiento de aguas servidas y, considerando que en esta enfermedad, a diferencia de otras entéricas, el ciclo corto de transmisión (persona a persona) es el preponderante. Una claro ejemplo de esto es el importante descenso experimentado por las tasas de hepatitis luego de la campaña masiva para la prevención del cólera en 1991. Por tal motivo, como respuesta a la actual epidemia, el Ministerio de Salud ha dado especial énfasis en la campaña de difusión de medidas de prevención. 


\section{Resumen}

Chile presenta un patrón epidemiológico de endemia media para hepatitis A con un desplazamiento paulatino de la edad de presentación de la enfermedad hacia la adolescencia y adultez. Desde 1975 al 2003 ha disminuido en 35\% la importancia relativa en niños bajo 10 años aumentando en $197 \%$ entre 15 y 24 años. Los ciclos epidémicos ocurren cada 3 a 5 años alcanzando tasas de 80 a 100 casos/100.000 hbtes. Durante el año 2003 y hasta la fecha se han notificado 8.263 casos con incidencia acumulada de 52,4 casos/ 100.000 y tasas máximas en los grupos 5 a 9 años (194/100.000) y 10 a 14 años (157/100.000). La distribución es a lo largo de todo el país y su presentación en brotes comunitarios, ocurriendo principalmente en grupos socieconómicos más bajos. Desde el año 2002 se ha reemplazado la inmunoglobulina humana por vacunación en el manejo de contactos. Actualmente se ha reforzado la campaña educativa para cortar el ciclo corto de transmisión y se trabaja en obras sanitarias para terminar con el ciclo largo.

\section{Bibliografía}

1.- WHO. Hepatitis A vaccines. Weekly Epidemiological Record 2000 Feb; 75 (5): 37-44.

2.- Abarca K, Vial P. Hepatitis A. En: Repetto G editor. Enfermedades Infecciosas Inmunoprevenibles. $1^{\circ} \mathrm{ed}$.
Santiago: Ediciones Universidad Católica de Chile; 2003: p 163-72.

3.- Centers For Diseases Control and Prevention. Prevention of hepatitis A through active or passive inmunization: Recommendations of the Advisory Committee on Inmunization Practices (ACIP). MMWR 1999; 48 (No RR-12): 9-18

4.- Departamento de Epidemiología, Ministerio de Salud de Chile. Situación de Enfermedades de Notificación Obligatoria: Hepatitis A. Boletín electrónico e-vigía 2003; $n^{\circ} 20$. Disponible en la world wide web en: http:/ /epi.minsal.cl/evigia/index.htm

5.- Departamento de Estadísticas e Información en Salud del Ministerio de Salud. Enfermedades de Notificación Obligatoria, Software Epivigil. Casos notificados hasta la semana epidemiológica $n^{\text {o }}$ 40: 28/09 al 04/10/2003.

6.- Medina B. Brotes de Hepatitis A en Chile. EneroAgosto de 2003. El Vigía 2003; 5 (18): 49-50.

7.- Sagliocca L, Amoroso P, Stroffolini T et al. Efficacy of hepatitis A vaccine in prevention of secondary hepatitis A infection: a randomised trial. Lancet 1999 Apr 3; 353 (9159): 1136-9.

8.- McMahon BJ, Beller M, Williams J, Schloss M, Tanttila H, Bulkow L. A program to control an outbreak of hepatitis A in Alaska by using an inactivated hepatitis A vaccine. Arch Pediatr Adolesc Med 1996 Jul; 150 (7): 733-9.

9.- Kaic B, Borcic B, Ljubicic M, Brkic I, Mihajjevic I. Hepatitis A control in a refugee camp by active inmunization. Vaccine 2001 14; 19 (27): 3616-9.

10.- Ibarra H, Riedemann S, Prado V et al. Estado actual de inmunidad para hepatitis por virus A en diversos grupos de adultos. Rev Méd Chile [online]. oct. 1999, vol.127, no.10 [citado 20 Octubre 2003], p.1165-8.

11.- Tello O, Amella C, Pachón Del Amo I, Martínez Navarro J F. Vigilancia de la Salud Pública. En: Martínez Navarro J F y cols. Salud Pública. $1^{\circ}$ ed. Ciudad de México: Mc Graw Hill; 1998: p 454-5.

Correspondencia a:

Bárbara Medina Daza

E-mail: bmedina@minsal.cl 